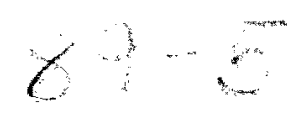

Comparison of Small Autonomous Robots by the Analysis of Their Functional Components

\author{
Peter W. Pachowicz \\ MLI 89.4 \\ 5
}




\title{
COMPARISON OF SMALL AUTONOMOUS ROBOTS BY THE ANALYSIS OF THEIR FUNCTIONAL COMPONENTS
}

\author{
Peter W. Pachowicz \\ Center for Artificial Intelligence \\ Computer Science Department \\ George Mason University \\ 4400 University Drive \\ Fairfax, VA 22030
}

\begin{abstract}
This report contains a brief review and analysis of several existing small autonomous robots in the U.S. This comprehensive study includes a description of their application area, sensing capabilities, computing facilities, degree of physical autonomy, and supporting software. Specific designing assumptions from this analysis have been presented for construction of our GMU Intelligent Explorer autonomous system.
\end{abstract}

\section{ACKNOWLEDGEMENTS}

The author wish to thank Professors Ken De Jong, Heedong Ko, Yves Kodratoff, Ryszard Michalski, Igor Mozetic, Jan Zytkow for their comments, and to thank Joyce Ralston for her help in the preparation of this report.

This work has been supported in part by the Defence Advanced Research Project Agency under grant, administrated by the Office of Naval Advanced Research Project Agency under grant No. N00014-87-K-0874, in part by the Office of Naval Research under grant No.N00014-88-K0226, and in part by the Office of Naval Research under grant No. N00014-88-K-0397. 


\section{INTRODUCTION}

Current AI progress and engineering sciences interests have created a common experimental research area for development of real-world intelligent systems. Such common interests run towards the increase of autonomy, i.e., the increase of self-decision making, understanding of surrounding environment, and self-adaptation to different tasks and variable environment. Following this direction, the AI Center at George Mason University has decided to create an experimental robot system. The system is specially designated to carry out currently developed experiments of the AI Center methodologies of machine learning, inference, automated reasoning, and planning. Our main goal is composed of the following two complementary parts:

- testing machine learning methodologies in increasing intelligent functions of autonomous robots,

- creation of an innovative adaptable autonomous robot, which is equipped with multiple sensors and actuators.

The designing step has been preceded by the specific analysis of several U.S. small autonomous systems with distinction of their hardware components. Section 2 presents such analysis according to our interests. Based on that analysis, Section 3 presents the main assumptions for the creation of our GMU-IEX autonomous robot. Finally, Section 4 summarizes this report.

The analysis has been based on the studies of the DARPA Research Programs, especially Image Understanding and Autonomous Land Vehicle programs research interests of primary investigators of the AI Center and other non-published materials.

\section{ANALYSIS OF SMALL AUTONOMOUS ROBOTS AND THEIR COMPONENTS}

The analysis presented in Section 2.2 has been limited to the consideration of existing small robot systems only. Such big systems as NAVLAB (CMU, Pittsburgh), and ALV (Martin Marietta, Denver) have been excluded due to their price, and our early phase experimental applications of machine learning methodologies. We assume that a small system is able to move in a typical indoor scene (i.e., office building with the crossing capabilities of door frames) and/or is able to move in limited area of an outdoor scene (e.g., 
pathways of the campus). Finally, we have selected the following four small systems of the U.S. robotics research scene:

- CMU-HERO robot, CMU - Pittsburgh (Simons, 1988);

- ALLEN, MOBOT-1/2 family, MIT - Cambridge (Brooks, 1988, Brooks et al., 1987);

- SRI robot, SRI - Menlo Park (Reifel, 1987);

- AuRA robot, University of Massachuset - Amherst (Arkin et al., 1987).

\subsection{Criteria for robot systems analysis}

Considering our stated goal, the analysis of these autonomous robot systems has been focused on their application, hardware components, complexity, limitations of physical autonomy, and software support. The analysis of robot internal control architectures has already been presented by Simmons (1988). He used the following criteria in his analysis: system decomposition, representation and connection of behaviors, planning and decisionmaking structures (for other comparison see also Rembold et al., 1987). Therefore, we will not analyze control layers and behaviors of robot systems. But, we will provide an engineering comparison of the robot hardware components, their mutual connections and applications. The analysis and comparison of those systems has been done in the following problem-oriented points of interests:

- APPLICATION DOMAIN --- Mobility (indoor, outdoor, inroom, cross-country),. object grasping, and object manipulation are basic functional criteria for robot comparison. They decide on robots' application areas and limitations of systems performance.

- SENSOR DATA FUSION --- Sensor equipment characterizes robot capability to acquire data from the environment. A great number of different types of sensors can improve the processes of the world perception, and can increase the robot's mental autonomy. At this point, we have studied all useful sensors of the compared robots as well as their location on the robot body.

- COMPUTING FACILITIES --- The evaluation of robot computer systems has taken into consideration the dislocation of computing hardware for on-board and off-board systems, and the specification of distinguished special hardware and standard 
computer equipment. Such analysis has been provided because of the real-time (or near-real time) requirements for robot performance.

- DEGREE OF PHYSICAL AUTONOMY --- We have studied robot physical autonomy because any physical link (such as cables between robot and external units) can limit not only the application domain of the autonomous systems, but also the capabilities of an experimental task execution. At this point, we have been interested in robots' connections with the external computer systems and power supply. In case of the elimination of physical cable-links, we have also analyzed the application of data radio-transmission.

- SUPPORTING SOFTWARE --- The analysis of robot software has included the choice of programming languages and trends in construction of such tools. We have specified software packages created particularly for: control of the robot mechanical units, processing of sensor data, high-level decision making, planning, reasoning, etc.

\subsection{The analysis of chosen robots}

The detailed characteristics of the chosen robot systems have been assembled in Appendix 1, while Table 1 presents a short comparison.

Studying this comparison and details from literature, one can see that the application domain of these robots has been limited generally to indoor mobility. However, the AuRA robot is able to operate in an outdoor natural scene, i.e., it is able to move through pathways with the distance limited by battery capacity and the power of UHF transmitter. All systems are laboratory vehicles created for the investigation of intelligent navigation and moving. Two of them (CMU-HERO robot, and the MIT robot family) have a additional on-board robot arm for simple object grasping and manipulation. This trend can suggest that the research teams are interested in the creation of more complex systems, where the cooperation between actuators is needed to perform complex tasks, instead of moving only. In such tasks, intelligent moving, manipulation, and grasping will be executed and coordinated in parallel.

In area of sensor data fusion, the robots use two separate sensor systems for navigation and world understanding. The first system uses black-and-white vision, which is accurate and gives more information about the environment using object-shape 
Table 1: Comparison of small autonomous robots

\begin{tabular}{|c|c|c|c|c|}
\hline & $\begin{array}{c}\text { CMU-HERO } \\
\text { robot }\end{array}$ & $\begin{array}{l}\text { ALLEN, } \\
\text { MOBOT1/2 }\end{array}$ & $\begin{array}{c}\text { SRI } \\
\text { robot }\end{array}$ & $\begin{array}{l}\text { AuRA } \\
\text { robot }\end{array}$ \\
\hline $\begin{array}{c}\text { Application domain } \\
\text { •mobility }\end{array}$ & indoor & indoor & indoor & indoor/outdoor \\
\hline $\begin{array}{l}\text { object grasping \& } \\
\text { manipulation }\end{array}$ & yes & yes & no & no \\
\hline $\begin{array}{l}\text { Sensor data fusion } \\
\text { - visual sensors }\end{array}$ & $\begin{array}{c}\text { 2-D b\&w off-board } \\
\text { vision }\end{array}$ & $\begin{array}{c}\text { calibrated stereo } \\
\text { b\&W vision }\end{array}$ & 2-D b\&w vision & 2-D b\&w vision \\
\hline - ultrasonic sensors & $\begin{array}{l}\text { rotated locator, } \\
\text { front obstacle } \\
\text { detector }\end{array}$ & ring of 12 sensors & ring of 12 sensors & ring of 24 sensors \\
\hline - tactile sensors & no & no & 8 bumpers & no \\
\hline $\begin{array}{c}\text { Computing facilities } \\
\text { - dislocation }\end{array}$ & on- and off-board & on-and off-board & on-board & on- and off-board \\
\hline - equippment & $\begin{array}{c}\text { on-board } \\
\text { microcomputer, } \\
\text { off-board WARP, } \\
\text { \& systems network }\end{array}$ & $\begin{array}{l}\text { on-board parallel } \\
\text { processors, off- } \\
\text { board Lispmachine }\end{array}$ & $\begin{array}{l}\text { on-board Z-80 } \\
\text { and SUN }\end{array}$ & $\begin{array}{c}\text { on-board } \\
\text { microcomputer, } \\
\text { off-board image } \\
\text { proc. and VAX }\end{array}$ \\
\hline $\begin{array}{l}\text { Degree of physical autonomy } \\
\text { - power supply }\end{array}$ & battery & battery & battery & battery \\
\hline - cable-links & for robot control & $\begin{array}{l}\text { for transmission } \\
\text { of images }\end{array}$ & no & no \\
\hline Computing software & $\begin{array}{l}\text { VANAGE, CODGER, } \\
\text { Framekit+, } \\
\text { 3-D FORM }\end{array}$ & N.A. & REX, CKS & $\begin{array}{l}\text { VISION, } \\
\text { GOLDIE }\end{array}$ \\
\hline
\end{tabular}


reconstruction from contour, texture, motion, and stereo. The second system, proximity sensing by ultrasonic sensors, works much faster than computationally expensive vision understanding, but it gives only a poor description of the environment. A specific lowpositioned ring of ultrasonic sensors on the robot body has been applied for moving among larger obstacles, in narrow indoor space, and along the walls. However, the CMU-HERO robot can detect obstacles by the front low-positioned ultrasonic sensor, which can be rotated with the entire robot platform around the system vertical axis. This solution is simpler than the creation of the sensors' ring but it requires additional search of sensed space by relatively much slower rotation of the robot body. Additionally, an over-robothead-rotated ultrasonic sensor (CMU-HERO) has been distinguished as a good solution for general navigation.

According to the dislocation of computing facilities, one can see only one system, i.e. the SRI robot, which computes all data by means of two on-board computers. In all other systems, main data processing and decision making is located off-board. Each robot possesses a lower-level controller for command execution and control of the data acquisition. Such controller is a single micro-computer of multi-purpose application, however, MOBOT 2 also possesses also distributed parallel processors for ultrasonic data processing and navigation. There is no significant off-board equipment type, which has been chosen commonly to work as a host system.

The analysis of the degree of physical autonomy of robots is a very strong common feature for all systems. Only battery supply has been used, in spite of the fact that several robots possess physical cable-connections with off-board computing facilities. For robots without on-board computer equipment (which is able to process images and other data received from sensor systems in a reasonable amount of time), it is necessary to create links with off-board computing systems. Two possible solutions have been used: cabletransmission and radio-transmission. The cable-transmission of data is the easiest solution for systems communication, but it highly limits the robot's physical autonomy. Such connection can be accepted only for the indoor (generally inroom) application domain during an experimental phase of system development. The second possibility, radio-transmission, can be applied as a digital serial-link for the robot control. If necessary, it can be used to transmit small amount of ultrasonic and tactile data, as well. But the serial connection cannot be applied for fast digital transmission of large images and RGB pictures. Considering the transmission of video signals by an UHF transmitter of the AuRA robot, one can distinguish such approach especially for the outdoor robot mobility. For the indoor 
mobility, one can only assume that high-frequency wave propagation will not be disturbed by the interference effects, industrial noise, and natural metal screens between transmitter and the receiver. However, we believe that the application of radio-transmission should be highly recommended for all robots independent on the external power supply. However, the final solution must consider the high increase of robot autonomy by the application of on-board high-speed computing facilities. In this area, the SRI robot is a good starting system for further development, however, the computing speed of SUN (located on the SRI robot body) cannot support sensor data fusion. Final application of small parallel processors is required.

The last element of our analysis, supported software, is not common for any two research teams because they have created individual data analysis and decision making software. However, most software and dedicated packages have been created on LISP or C language basis. The general tendency is to create and use object-oriented software.

\subsection{Summary of the analysis}

In summary, one can distinguish the SRI robot, which is really independent on power and computing support. Unfortunately, it cannot perform object grasping and manipulation, and it works relatively slowly because of the slow image data processing supported by the SUN computer. The second system, the AuRA robot, possesses similar physical autonomy, however, the quality of the very fast off-board image analysis and decision making depends highly on the quality of UHF image radio-transmission. According to the stereo-vision applied on the MIT family robots, there are physical cable-connections of two cameras with off-board image computing system. Technical radio-transmission of multiimages requires extension of the transmission system from the simplest "master-slave" mode to the "handshaking" transmission mode. One must point out that the CMU-HERO autonomous robot uses off-board cameras. But, the robot perception capabilities in such approach are extremely limited, and the robot mobile autonomy (really limited by the number of cameras and their dislocation) is difficult to understand in this comparison. Such approach also excludes advantage experimentation in the area of new methodologies to the robot sensing, i.e., active vision (Aloimonos and Shulman, 1987, Aloimonos et al., 1987), vision with feedback (Bajcsy, 1988), and adaptable sensing (Pachowicz, 1988). 


\section{DESIGNING ASSUMPTIONS FOR THE CREATION OF GMU INTELLIGENT EXPLORER}

This section presents our defined requirements for designing a new small autonomous robot system with extended mental and perceptual autonomy. We assume that the mental autonomy of a robot is the capability to learn, discover, and make decisions itself within a dynamic environment. Perceptual autonomy is a robot's capability to create and change sensing strategies, both within a single sensor and also for multiple sensors integration. We can create self-adaptation of sensor devices and their data understanding modules to a new task (e.g., location, recognition, inspection), known/unknown objects, and external conditions (e.g., noise, light sources, etc.). The requirements presented below have been grouped and discussed according to our interests and the above analysis of small autonomous robots.

\section{Primary goal assumption}

- A new robot system must operate in the environment to support experiments with high-level AI (i.e. machine learning, inference, planning, reasoning, and other methodologies under development at the AI Center). One expects to test the usefulness of $\mathrm{AI}$ in the extension of robot intelligence, the increase of robot perceptual capabilities, performance improvement of actuators, and the creation of self-adaptability mechanisms.

\section{Assumptions of our secondary interests}

- Provide an extension of the number of sensors, the sensing capabilities of a single sensor, and integrate multi-sensors into a flexible robot perception system.

- Increase operational capabilities of the robot to perform such functions as moving, object grasping, and object manipulation, all executable in parallel.

- Create self-adaptation capabilities of the robot and its subsystems to a new environment and a new task.

- Investigate an application of new modern approaches to robot perception, i.e. active sensing, sensing with feedback, adaptable perception. 
Assumptions dealing with previous analysis of small autonomous robots

- Limit the application domain to indoor mobility (temporary inroom mobility) and focus on the integration of $\mathrm{AI}$ and robotics;

- Build a great number of sensors for visual, proximity, and tactile sensing on the robot body. Create an integration of data from multiple sensors, where the same features of environment (e.g., geometrical structure of objects) can be perceived by a set of different sorts of sensors instead of the use of only one sensor;

- Locate the color camera on the robot arm, and develop methodologies for the camera guidance. Create an intelligent visual sensing system capable of detecting shapes from color, texture, contour, motion, and multi-view directions.

- Use various off-board distributed computing facilities, and create relocable software with well specified interfaces and communication protocols. Consider fast parallel technologies (e.g., application of transputers - see Bakkers and Verhoeven, 1987) for on-board hardware computation in future system implementation.

- Create the robot system using the battery supply and desirable physical cable-links between the robot body and external computing facilities. Predict the possibility to apply radio-transmission for the final system application (e.g., nuclear or military plant robot);

- Use object-oriented programming tools, and create own programming packages as a subject for technology transfer and cooperation.

\section{Additional technical assumptions}

- We minimize the direct handmade engineering work, and we complete the system from manufactured boards and current computer and robot facilities of the AI Center (Stefanski, 1988).

- In the first step of the research work, we focus on the creation of the robot's mental and perceptual autonomy (i.e., realized by software), instead of the development and extension of robot physical autonomy. 
- Everyone of the AI Center expects to work with the system, which is easily managed and functionally ready to support AI tasks and individual software.

\section{CONCLUSIONS}

This report has presented the review and analysis of four chosen small autonomous robots of the US robotics and AI scene. Those robots have been created for intelligent navigation and moving. However, the creation of the CMU-HERO robot has been caused by experimentation with machine learning in area of simple robot operational functions (e.g., object delivery). Unfortunately, that system has very limited perceptual autonomy, and we conclude that there was no intention to apply machine learning methodologies within sensory systems. Other groups have spent a lot of effort on the investigation of the visual world perception with application to robot navigation. Vision sensing has been based on the black and white image, mono or stereo vision, and motion. They also worked on the increasing the physical autonomy of their robots, and the development of special software packages.

Considering presented analysis of these four small autonomous robots, we have created a list of main assumptions for building our GMU-IEX autonomous robot. This list has been completed with additional assumptions derived from our interests and technical reasons. Studying that list of assumptions, one can mention that our approach for the creation of an intelligent autonomous robot differs from approaches of the other research groups. We need to test specific tools of machine learning. Then, the creation of our autonomous robot system is just guided by the development of AI, while other research groups seem to work on robotics domain with searching AI tools. In our case, the increase of GMU-IEX physical autonomy is less important than the increase of mental and perceptual autonomy. 


\section{REFERENCES}

Aloimonos, J.Y. and Shulman, D., "Learning Shape Computations". Proc. DARPA Image Understanding Workshop, Los Angeles, 1987, pp.862-873.

Aloimonos, J.Y., Weiss, J. and Bandyopadhyay, A., "Active Vision". Proc. DARPA Image Understanding Workshop, Los Angeles, 1987, pp.552-573.

Arkin, R.C., Riseman, E.M. and Hanson, A.R., "AuRA: An Architecture for VisionBased Robot Navigation", Proc. DARPA Image Understanding Workshop, Los Angeles, $1987, \mathrm{pp} .417-431$.

Bajcsy, R., "Perception with Feedback". Proc. DARPA Image Understanding Workshop, Cambridge, 1988, pp.279-288.

Bakkers, A.P. and Verhoeven, W.L.A., "A Robot Control Algorithm Implementation in Transputers, Proc. Intelligent Autonomous Systems, L.O.Hertzberg and F.C.Groen (Ed.), Amsterdam 1986, North-Holland, 1987, pp.118-122.

Brooks, R.A., Flynn, A.M. and Marill, T., "Self Calibration of Motion and Stereo Vision for Mobile Robot Navigation", Proc. DARPA Image Understanding Workshop, Cambridge, 1988, pp.398-410.

Brooks, R.A., "A Robust Programming Scheme for a Mobile Robot", in Languages for Sensor-Based Control in Robotics, U.Rembold and K.Hormann (Ed.), NATO ASI Series, vol.F29, Springer Verlag, 1987, pp.509-522.

Pachowicz, P.W., "Local Characteristics of Binary Image for Potential Automatic Choice of Algorithms in Low-Level Vision", submitted to Computer Vision, Graphics, and Image Processing, 1988.

Reifel, S., "The SRI Mobile Robot Testbed", SRI Technical Note 413, 1987.

Rembold, U. and Levi, P., "Sensors and Control for Autonomous Robots", Proc. Intelligent Autonomous Systems, L.O.Hertzberg and F.C.Groen (Ed.), Amsterdam 1986, North-Holland, 1987, pp.79-89.

Simons, R., "Mobile Robot Architecture", seminar material, 1988.

Stefanski, P.A., "An Introduction to the Computer Facilities of the GMU Center for Artificial Intelligence", Technical Report of the Center for AI, George Mason University, TR-16-88, 1988. 


\section{APPENDIX 1: CHARACTERISTICS OF SELECTED ROBOT SYSTEMS}

\section{CMU-HERO robot:}

Application domain

- typical indoor navigation and moving,

- object grasping and manipulation with imprecise positioning;

Sensor data fusion

- visual sensing by numerous stable cameras mounted above the roof of the laboratory rooms/hallways,

- proximity sensing by rotated overhead ultrasonic sensor, single over-floor front detector of obstacles;

Computing facilities

- small on-board microcomputer, WARP off-board fast parallel processor and other computers of the network;

Physical autonomy

- battery supply for all on-board subsystems,

- single fast serial cable-link or slow radio-transmission between HERO robot and off-board computers;

Supporting software

- VANAGE frame-based geometrical modeling system, Framekit+ knowledge representation system, 3-D FORM geometric reasoning system, CODGER central geometric database for putting all sensor data together.

\section{ALLEN, MOBOT-1/2 family:}

Application domain

- indoor navigation and moving with distinction of obstacle avoidance,

- object grasping and manipulation,

Sensor data fusion

- calibrated on-board stereo vision (black and white),

- ring of 12 ultrasonic sensors;

Computing facilities

- on-board parallel processors for sonar data analysis; offboard computing power for vision support and robot control (Lisp machines);

Physical autonomy

- battery supply for all on-board subsystems,

- cable-connections of stereo vision cameras with off-board processing system, cable-link for robot control; 


\section{SRI - robot:}

Application domain

Sensor data fusion

Computing facilities

Physical autonomy

Supporting software
- typical indoor (rooms and hallways) navigation and moving, possible outdoor pathways following and moving;

- on-board 2-D black and white camera calibrated by hand,

- ring of 12 ultrasonic sensors,

- eight tactile bumpers,

- on-board Z-80 microcomputer, and SUN system with harddisc, off-board Lisp-machine for automatic generation of SUN's processing software;

- battery supply for all on-board systems;

- no-cable links with off-board Lisp machine, there has been applied radio-transmission of program and data;

- REX Lisp-based programming language for building complex machines by declarative description of their behavior, CKS - Core Knowledge Structure to support new generation of knowledge-based generic vision systems.

\section{AuRA robot:}

Application domain

Sensor data fusion

Computing facilities

- indoor (hallways) and outdoor navigation and moving;

- 2-D black and white camera with image radio-transmission;

- ring of 24 ultrasonic sensors;

- on-board controller of moving (in plans, 12 node multiprocessor to execute distributed specific algorithms that can work in parallel), off-board VAX 11/750 and image processing system;

Physical autonomy

- battery supply for all on-board subsystems,

- no-cable links, images are transmitted by on-board UHF transmitter,

Supporting software

- VISION software system for mobile robot navigation, GOLDIE top-down controller of low- and intermediate-level image understanding processes. 